\title{
The Benefits of Mindfulness for University Students
}

\author{
Christina Maher, BS \\ Fairfield University
}

\begin{abstract}
Background: Mindfulness training is increasingly being adopted to address mental health concerns among university students (Kuyken et al., 2017). Previous research has supported mindfulness with empirical evidence as an autonomous, accessible, and cost-effective intervention for stress and anxiety (Flett, Conner, Rordan, Patterson, \& Hayne, 2020).

Aim: The purpose of this study was (1) to examine the prevalence of student stress and anxiety alongside student mindfulness interest and experience; (2) to examine the benefits of mindfulness training through the perspectives of cognitive neuroscience, population health, and behavioral economics; and (3) to examine the cost-effectiveness of mindfulness training for university students.

Methods: To address these aims, an anonymous online survey incorporating both the Five Facet Mindfulness Questionnaire (FFMQ) and the Perceived Stress Scale (PSS) was administered to students at a liberal arts university in New England to quantify the prevalence of student stress and mindfulness. It was hypothesized that scores on the PSS would be inversely correlated with scores on the FFMQ .

Results: The results demonstrated a significant negative correlation between FFMQ and PSS scores, $r$ $(107)=-.507, p<0.01$.

Conclusion: The implications of this study are beneficial in establishing mindfulness training as an effective and cost-effective approach to promoting mental health in students. The study concluded with a data-informed recommendation for educational strategy based upon survey and research findings.
\end{abstract}

Submitted 01 July 2020: accepted 16 January 2021

Keywords: student, mental health, mindfulness, university

A growing body of evidence demonstrates a significant correlation between mindfulness practice and a reduction in maladaptive cognitive and behavioral functions commonly associated with mental health disorders (Basso, McHale, Ende, Oberlin, \& Suzuki, 2018; Taylor, Strauss, Cavanagh, \& Jones, 2014; Kuyken et al., 2017). Ensuring accessibility, efficiency, and efficacy of mental health resources should be a priority of universities, as academic success and student retention depends upon mental health (Lipson, Abelson, Ceglarek, Phillips, \& Eisenberg, 2019; Eisenberg, Lipson, \& Posselt, 2016). Both the demand and complexity of needs for mental health services are exceeding capacity as university student enrollment and diversity are increasing globally (Duffy et al., 2019). One in three college students report experiencing depressive symptoms with maladaptive behavioral implications impeding everyday functioning. Of these students, 4\% report having seriously considered suicide. Among people age 18-24, half of all health concerns are related to mental health disorders (Hunt \& Eisenberg, 2010). Additionally, 75\% of mental health disorders manifest before the age of 24 (Kessler, Berglund, Demler, Jin, \& Walters, 2005 as cited by Kuyken et al., 2017). 
The etiology of many mental health disorders among university students may be attributed to the first year, as it is a period of heightened transition, change, and adjustment (Dogan, 2012; Flett et al., 2020). The present study sought to examine the benefits of mindfulness as a prophylactic intervention to enhance mental health, wellbeing, and socialemotional behavioral functioning for students. This study employed a population health approach in studying the feasibility, acceptability, cost-effectiveness, and neurocognitive and behavioral implications of mindfulness training as a potential augmentation to a universal university curriculum program. Therefore, this study sought to ascertain mindfulness's potential benefits for the whole population, not just those at high risk (Kuyken et al., 2017). The primary aims of the present study were to conduct exploratory research examining (1) prevalence of student stress and anxiety alongside student mindfulness interest and experience; (2) the benefits of mindfulness training through the perspectives of cognitive neuroscience, population health, and behavioral economics; and (3) the cost-effectiveness of mindfulness training for university students.

\section{BACKGROUND}

\section{Cognitive Neuroscience of Mindfulness}

Mindfulness is defined as a state of moment-to-moment awareness of one's experience without judgement (Davis \& Hayes, 2012). Mindfulness training involves systematic and sustained focused attention (Kuyken et al., 2017). Studying mindfulness through the lens of cognitive neuroscience allows for deeper understanding of the connection between the mind and brain. Mindfulness training is associated with decreased rumination, a common symptom of depression, and enhanced attention (Wolkin, 2015). Mindfulness training has been associated with increased brain activity in the prefrontal cortex and hippocampus, two regions associated with attention (Basso et al., 2018; Wheeler, Arnkoff, \& Glass, 2017). Basso et al. (2018) findings suggested significant mindfulness-induced changes in emotional regulation and affect among a sample of non-experienced mindfulness practitioners who completed 8 weeks of brief daily mindfulness practice (Basso et al., 2018). Basso et al. (2018) findings further suggested that a relatively short daily mindfulness practice has similar cognitive results as longer duration and higher-intensity mindfulness practice in nonexperienced participants. These findings are particularly relevant as the present study sought to investigate mindfulness as an efficient and effective intervention for students.

Wheeler et al. (2017) reported that behavioral differences in attention and psychological well-being were positively correlated with structural differences in the brain's emotional regulation network for mindfulness practitioners. Greater amygdala volume is suggested to be associated with greater cognitive control of emotional responses. The neuroplastic effects of mindfulness training have been associated behaviorally with enhanced cognitive task performance and self-reported reduction in depression, anxiety, and psychopathology symptomatology (Basso et al., 2018; Bostic et al., 2015; Farb et al., 2010; Galante et al., 2018). Investigating the neural underpinnings of mindfulness helps researchers understand its associations with behavior, emotion, and cognition. This allows for mindfulness to be integrated into targeted therapeutic techniques and education strategy.

\section{Mindfulnomics: Behavioral Economics \& Mindfulness}

Previous research has suggested that interventions based upon self-determination theory (SDT), which states that humans strive for a unified sense of self within a framework of social inclusion, have efficacy in uptake and retention of positive health-related behaviors and promoting adaptive physiological outcomes (Deci \& Ryan, 1985; Teixeira et 
al., 2020). SDT, first proposed by Deci and Ryan (1985), addresses intrinsic goal-orientated motivation and the factors which drive motivation and influence our well-being and development. SDT promotes that health behavior change is most successful when the intended behavior fulfills three basic psychological needs for autonomy, competence, and relatedness (Ryan \& Deci, 2000). The need for autonomy refers to the experience of ownership and responsibility for one's own actions. The need for competence refers to feeling competent and capable to master tasks. Finally, the need for relatedness refers to a sense of connectedness and acceptance (Ryan \& Deci, 2000). Autonomous and intrinsic goal setting are constructs of mindfulness and postulates of the self-determination theory (Schultz \& Ryan, 2015). Awareness and self-reflection are promoted by mindfulness and serve as integral components of autonomy development. Brown and Ryan (2003) found that mindfulness was positively correlated with the basic needs described by SDT (autonomy, competence, and relatedness). Further, mindfulness in students was positively correlated with self-reported feelings of autonomy and mood regulation. The mechanistic pathways through which mindfulness works include, decreasing automatic maladaptive behaviors, promoting open attention and awareness alongside the detachment from ego-centric biases and reactivity, and emphasizing intrinsic values of community involvement and lower materialism (Brown \& Ryan, 2003).

Charbonneau (2019) studied the correlates between behavioral economic's theory of psychological need fulfilment and mindfulness among a group of military college cadets. Results suggested that teaching mindfulness to future military officers was beneficial for enhancing resilience and fulfilling their basic psychological needs for autonomy, competence, and relatedness. This need fulfillment was associated with enhanced psychological well-being among military cadets (Charbonneau, 2019).

Mindfulness has also been cited for its potential to reduce the effects of cue-induced craving on alcohol demand and consumption among university students (Hochster, Block-Lerner, Marks, \& Erblich, 2018). Student drinking is an issue on campuses as 39\% of students partake in binge-drinking, according to the 2014 National Survey on Drug Use and Health (Hochster et al., 2018). University drinking is associated with poorer academic performance and retention, higher rates of sexual assault, injury, and the development of alcohol use disorders. Hochster et al. (2018) results demonstrated that mindfulness buffered the behavioral economic demand for alcohol among a sample of college drinkers who completed a laboratory-based cue-induced craving assessment, a self-report assessment of trait mindfulness, and an alcohol purchase task.

\section{Student Mental Health \& Mindfulness}

Previous research has suggested a significant correlation between mindfulness and emotional regulation (Kuyken et al., 2017). Maladaptive emotional regulation has been linked to more than $50 \%$ of Axis I and $100 \%$ of Axis II disorders listed in the Diagnostic and Statistical Manual of Mental Disorders (Gross \& Levenson, 1997 as cited in Wheeler et al., 2017). Previous research has studied the implications of mindfulness for students (Bostic et al., 2015; Dunning et al., 2018; Flett et al., 2020; Galante et al., 2018; Kuyken et al., 2017; Lavelle Heineberg, 2016; Meiklejohn et al., 2012; Riet, Levett-Jones, \& Aquino-Russel, 2018; Taylor et al., 2014;). University students completed an 8-week mindfulness course and demonstrated improved resilience to stress during their examination period, according to scores on the Clinical Outcomes in Routine Evaluation Outcome Measures (Galante et al., 2018). Mindfulness has been found to mitigate nursing students' stress, anxiety, depression, and burnout while promoting a sense of well-being and empathy (Reit et al., 2018). Shapiro, Schwartz, and Bonner (1998) found that medical students who received mindfulness training displayed heightened empathy, even under heightened stress when compared to controls. Mindfulness has 
been found to help students develop emotional regulation, conflict resolution, and resilience strategies. Bostic et al. (2015) demonstrated that short duration mindfulness training induced neurobiological adaptations in student stress responses.

Dunning et al., (2018) conducted a meta-analysis examining the behavioral, cognitive, and mental health outcomes for students following a mindfulness intervention. Their results demonstrated significant positive effects of mindfulness-based interventions for outcomes of mindfulness, executive functioning, attention, depression, anxiety, stress, and maladaptive behaviors. Flett et al. (2020) studied the impact of an app-based mindfulness meditation upon psychological distress and college adjustment in incoming university students. Their findings demonstrated that participants who used the app more frequently reported improvements in psychological distress and college adjustment. These findings are particularly relevant to the present study as they suggest in-person mindfulness interventions delivered at the beginning of the academic year for first-year students may encourage uptake and retention.

Interventions based upon mindfulness-based stress reduction and mindfulness-based cognitive therapy include weekly group practice, a curriculum of basic intentional mindfulness exercises (body scan, sitting, movement, and walking meditations), and an emphasis upon the recruitment of dispositional mindfulness towards everyday experiences (Bostic et al., 2015). These interventions have been specifically adapted for students, called Mindfulness Skills for Students (MSS). Previous research has associated MSS with social, emotional, and prosocial student development (Kuyken et al., 2017; Lavelle Heineberg, 2016; Meiklejohn et al., 2012). Further, self-help mindfulness courses, which promote student autonomy and independent skill-building to address mental health concerns, are a low-cost and readily available intervention which have demonstrated significant improvements in measures of depression, anxiety, stress, life satisfaction, mindfulness, and self-compassion among students (Taylor et al., 2014).

Student mental health initiatives are beneficial to both a university's academic mission and its economic success (Lipson et al., 2019). Lipson et al. (2019) recommended that universities prioritize mental health through population health-focused, campus-wide, and curriculum-based programs. Herman et al. (2017) evaluated the cost-effectiveness of Mindfulness-Based Stress Reduction (MBSR) compared to Cognitive Behavior Therapy (CBT) from a societal perspective by calculating the incremental sum of healthcare costs and productivity losses over change in qualityadjusted life-years (QALYs). Results demonstrated that the mean incremental cost per participant to society of CBT was $\$ 125$ whereas MBSR produced a net savings of $\$ 724$ (Herman et al., 2017). Additionally, Feliu-Soler et al. (2018) demonstrated acceptable cost-effectiveness and cost-utility ratios for MBSR.

The present study's aims were (1) to examine the prevalence of student stress and anxiety alongside student mindfulness interest and experience; (2) to examine the benefits of mindfulness training through the perspectives of cognitive neuroscience, population health, and behavioral economics; and (3) to examine the cost-effectiveness of mindfulness training for university students. Based on previous findings, it was hypothesized that perceived stress would be inversely correlated with mindfulness. The study concluded with a data-informed recommendation for education strategy, based on the present study's survey and literature review findings regarding the prevalence of stress among students and both the cost- effectiveness and neurocognitive benefits of mindfulness training.

\section{METHODS}

The study's aims were addressed using an anonymous online survey administered to students at a liberal arts university in New England. Through this survey, students were administered the FFMQ (Karl et al., 2020) and the Perceived 
Stress Scale (PSS; Medvedev et al., 2019) to ascertain the prevalence of and potential correlation between student selfreported mindfulness and stress.

\section{Five Facet Mindfulness Questionnaire}

The version of the FFMQ administered in this study is a variation of the FFMQ developed by Baer et al. (2006) at Kentucky University. The FFMQ measures the five aspects of mindfulness. These include observation, description, aware actions, non-judgmental inner experience, and non-reactivity (Baer et al., 2006). Observation refers to the way one sees, feels, and perceives the internal and external world using their sensory awareness. An example of an FFMQ question which measures observation is, "when I'm walking, I deliberately notice the sensations of my body moving." Description refers to the ways in which one labels an experience and relays the experience in words to oneself and others. An example of an FFMQ question which measures description is, "I'm good at finding words to describe my feelings." Aware actions refers to the movements we choose after attending to the information present at the moment. An example of an FFMQ question which measures aware actions is, "when I do things, my mind wanders off and I'm easily distracted." Non-judgmental inner experience refers to self-acceptance and unconditional empathy for oneself and others. An example of an FFMQ question which measures non-judgmental inner experience is, "I criticize myself for having irrational or inappropriate emotions." Finally, non-reactivity refers to active detachment from negative thoughts and emotions. An example of an FFMQ question which measures non-reactivity is, "I perceive my feelings and emotions without having to react to them." (Baer et al., 2006).

Participants responded on a 5-point Likert scale (never/very rarely true, rarely true, sometimes true, often true, or very often/always true) to a series of 39 questions. Participants were instructed to select a response that best describes their own opinion of what is generally true for them. Previous research has associated the FFMQ with high construct validity as FFMQ scores were significantly related to experience of formal mindfulness practice, psychological symptoms, and wellbeing (Baer et al., 2008; Bohlmeijer, Ten Klooster, Fledderus, Veehof, \& Baer, 2011). For reliability, prior studies reported the FFMQ's internal consistency estimates (Cronbach's alpha) ranging from 0.75 to 0.93 (Christopher, Neuser, Michael, \& Baitmangalkar, 2012; Baer et al., 2008; Shallcross, Lu, \& Hays, 2020). A Cronbach's alpha of 0.70 is considered the minimum measure for internal reliability (Nunnally \& Berstein, 1994).

\section{Perceived Stress Scale}

The PSS measures the degree to which one's life situations are perceived as stressful. One's perception is most important for this survey. Two individuals with the same life experience in the past month may present two different scores, one in the low stress category and the other in the high stress category. This variation is dependent upon the individuals' perception, resilience, and coping mechanisms. The version of the PSS administered in this study is a variation of the PSS developed by Cohen, Kamarck, and Mermelstein (1983). Participants are asked general questions about their current levels of stress rather than focusing upon specific experiences.

Participants were asked to report their feelings and thoughts during the last month, responding to 10 questions in accordance with a 5-point Likert scale (never, almost never, sometimes, fairly often, or very often) regarding how often they felt or thought a certain way. Some examples of questions asked on the PSS are, "In the last month, how 
often have you been upset because of something that happened unexpectedly?"; "In the last month, how often have you felt that things were going your way?"; and "In the last month, how often have you been angered because of things that were outside of your control?" (Cohen et al., 1983). Summation of all the direct and reverse items equate to the participant's overall PSS score. Convergent validity of the PSS has been supported by previous studies which demonstrated positive correlation between PSS scores and scores on measures of mental and physical health (Baik et al., 2019). Baik et al. (2019) demonstrated that participants who scored highly on the PSS also scored highly on measures of anxiety and depression symptom severity. For reliability, previous studies have reported PSS's internal consistency estimates (Cronbach's alpha) to be greater than 0.70 (Lee, 2013).

\section{Procedure}

Participants were recruited using undergraduate social media pages (i.e., Facebook). A link to the survey was posted on the university's first-year, second-year, third-year, and fourth-year student Facebook pages. Prior to recruitment, the survey protocol was approved by the university's Institutional Review Board (IRB). Only undergraduate students were recruited, and participants of any academic major were included. Participant responses were associated with a random number and stored using Qualtrics, a cloud-based software platform for surveys. Participants were required to provide informed consent prior to participating in the study. Participants were informed that their demographic information and survey scores would be anonymous. Participant names or other identifying information were not collected, and all responses were associated with a random number assigned to each participant by Qualtrics.

First, students responded to a series of demographic questions. Participants were asked to provide their academic year, gender, and whether or not they would have been interested in learning about mindfulness during their mandatory first year orientation course. Participants then proceeded to complete the FFMQ and PSS. A literature review was conducted on Psycinfo using the following search terms, "student mental health," "mindfulness," "student mindfulness," "cognitive neuroscience of mindfulness," "behavioral economics of mindfulness." Articles which addressed the implications of mindfulness for student mental health, the behavioral economics of mindfulness, and the cognitive neuroscience of mindfulness were included. The findings of the present study were evaluated in light of previous findings.

\section{Data Analysis}

Descriptive statistics were used to examine sample demographics and participants' responses to the FFMQ and PSS. Pearson's correlation coefficient was calculated using SPSS software to determine a potential relationship between participant scores on the FFMQ and PSS. Pearson's correlation coefficient was conducted because a linear relationship was hypothesized between scores on the FFMQ and PSS. Outliers were identified as FFMQ and PSS scores falling above the upper range limit $(\mathrm{Q} 3+[1.5 * \mathrm{IQR}])$ or below $(\mathrm{Q} 1-[1.5 * \mathrm{IQR}])$.

Additionally, a linear regression was conducted to determine if participant responses to the question, "Would you have been interested in learning about mindfulness during your First Year Experience course?" was a significant predictor of FFMQ and PSS scores. 


\section{RESULTS}

Participants included 111 undergraduate students enrolled at a liberal arts university in New England. Participants were first-year $(n=7)$, second year $(n=11)$, third year $(n=12)$, and fourth year $(n=81)$ students. The sample included both female $(n=88)$ and male $(n=23)$ participants. Participants' openness to mindfulness was collected, and participants reported either yes $(n=90)$, no $(n=3)$, or maybe $(n=18)$ for whether they would have been interested in learning about mindfulness during their mandatory first year orientation course. There were two scores determined to be outliers, and these participants were excluded from analysis $(n=109)$.

The means and standard deviations of participant scores on the FFMQ were $\mathrm{M}=122.12, \mathrm{SD}=16.24$. The means and standard deviations for the PSS were $\mathrm{M}=32.90, \mathrm{SD}=6.30$. Pearson's correlation coefficient was used to assess the relationship between participant mindfulness and stress. There was a significant negative correlation between FFMQ and PSS scores, $r(107)=-0.507, p<0.01$. This negative correlation suggested that as FFMQ mindfulness scores increased, PSS stress scores decreased. A participant who scored high in FFMQ mindfulness, scored low in PSS stress. The inverse is true for participants who scored low on FFMQ mindfulness.

A linear regression was conducted to determine if participant responses (yes $=1$, maybe $=2$, no $=3$ ) to the question, "Would you have been interested in learning about mindfulness during your First Year Experience course?" significantly predicted FMMQ scores. A significant regression equation was found $\mathrm{F}(1,107)=4.44, \mathrm{p}<0.05, \mathrm{R}^{2}=$ 0.04). Participants' predicted FFMQ scores were equal to $130.05+\mathrm{x}(-6.39)$ where $\mathrm{x}=1$ (yes), 2 (maybe), 3 (no). Participants' predicted FFMQ scores were equal to 124.40 when they responded "yes" and 110.88 when they responded "no." This finding suggested that there was a relationship between participants who expressed their interest in having learned about mindfulness during their First Year Experience course and their self-reported mindfulness when completing the FFMQ. This relationship further predicted that participants who responded affirmatively to this question had higher FFMQ mindfulness scores than those who expressed their disinterest in having learned about mindfulness during their First-Year Experience course. However, the low proportion of variance accounted for by this model, $\mathrm{R}^{2}=0.04$, suggested a high amount of unexplained variability in FFMQ scores not predicted by participants' responses to the question, "Would you have been interested in learning about mindfulness during your First Year Experience course?" 


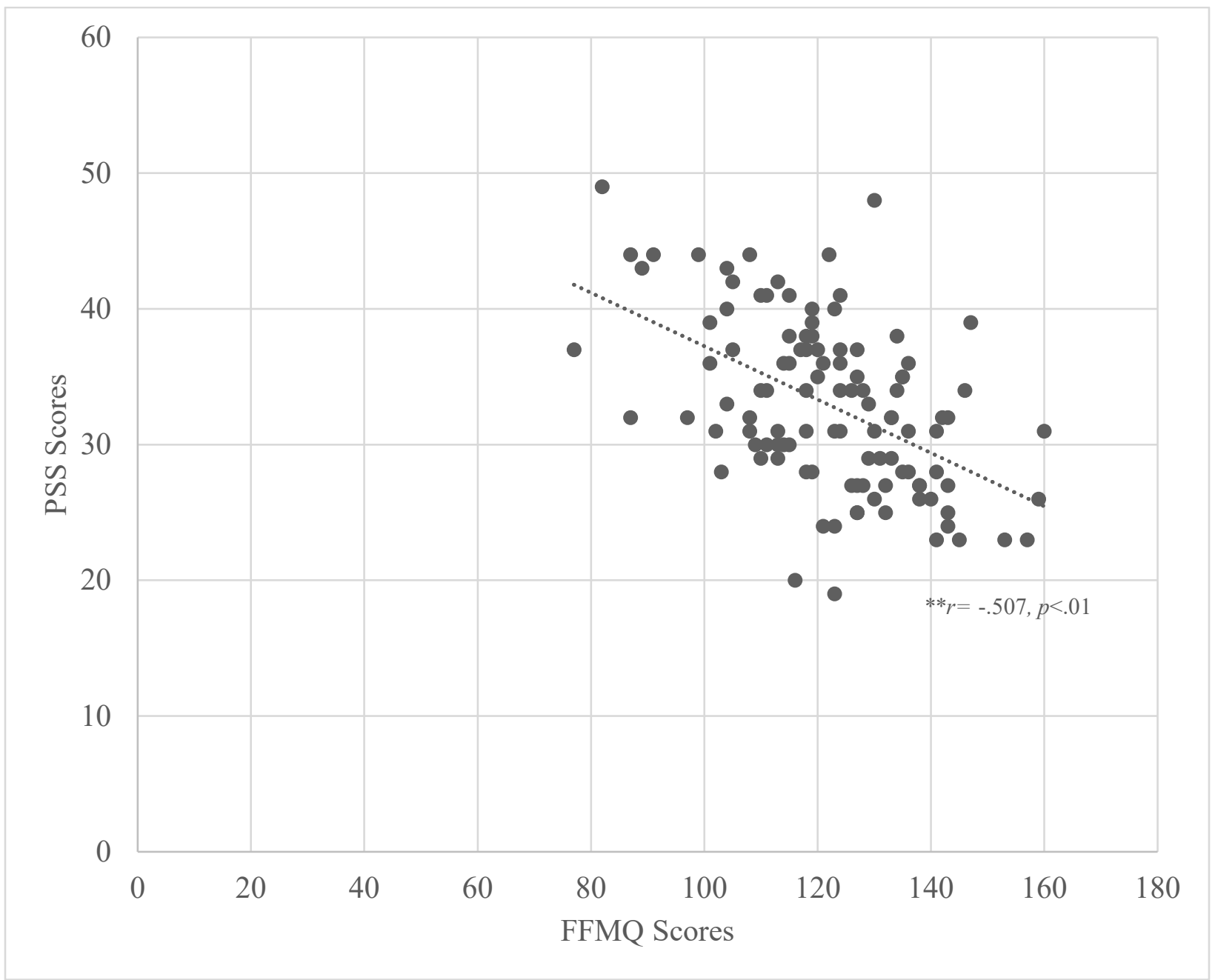

Figure 1. The negative correlation between FFMQ and PSS scores is depicted, $r(107)=-0.507, p<0.01$. As scores on the FFMQ (depicted on the $\mathrm{x}$ axis) increased, scores on the PSS decreased (depicted on the y axis). Two outliers were removed from analysis and were not depicted $(n=109)$. These findings supported the hypothesis.

\section{DISCUSSION}

A statistically significant negative correlation was found between participant's mindfulness scores on the FMMQ and stress scores on the PSS. These findings support the hypothesis that scores on the PSS are inversely correlated with scores on the FFMQ and reflect previous research which has suggested that a negative correlation exists between mindfulness and perceived stress (Baer, Carmody, \& Hunsinger, 2012). This negative correlation suggested that participants who had high FFMQ mindfulness scores had low PSS stress scores. As FFMQ mindfulness scores increased, PSS stress scores decreased. Additionally, $82 \%$ of participants responded that they would have been interested in learning about mindfulness during their mandatory First-Year Experience course. This result suggested general acceptability and interest among students regarding the augmentation of mindfulness to university curriculum. This finding is reflected in previous research which associated mindfulness programs with high acceptability and retention (Minkler et al., 2019). 
Participants' response regarding their interest in having learned about mindfulness during their First-Year Experience course was a significant predictor of FFMQ scores, with affirmative responses predictive of higher FFMQ scores. This question reflected similar questions asked in previous literature to ascertain the acceptability and feasibility of mindfulness training for students (Dvořáková et al., 2017). This finding may have reflected a potential predisposition of participants towards mindfulness. However, it is important to note that the independent predictor variable (participants' question responses) in this model accounted for a small proportion of variance $\left(\mathrm{R}^{2}=0.04\right)$, or variability, in the dependent variable (FFMQ scores). The inclusion of additional independent predictor variables like, PSS scores or demographic characteristics, may have strengthened the model's ability to predict FFMQ scores. Students without prior experience of mindfulness may have been unclear regarding what "learning about mindfulness" entailed. Participant responses were not a significant predictor of PSS scores. PSS scores ranging between 27 - 40 are considered high perceived stress. The average PSS score for the present study was within the range of high perceived stress $(\mathrm{M}=32.90 ; \mathrm{SD}=6.30)$. These findings reflect those of Kwok and $\mathrm{Ng}(2016)$ which associated university students with higher-than-average scores of perceived stress according to the PSS. Both findings are indicative of a need to develop educational strategy which promotes mental health resilience and healthy coping mechanisms.

For demonstrative purposes, the return on investment (ROI) for a university implementing mindfulness training was calculated using the Healthy Mind's Network ROI Calculator for College Mental Health Resources (Lipson et al., 2019). The calculation was based upon research examining the implications of depressive symptoms for student performance and retention. Approximate numbers were provided regarding student population size, tuition rate, program costs, and attrition rate. University return on investment was calculated as a function of these variables. According to the ROI Calculator, the implementation of mindfulness training would result in approximately 62 students retained annually, approximately $\$ 6.2$ million added tuition revenue from retained students, and approximately $\$ 12.3$ million added lifetime earnings for retained students based upon increased societal productivity (Eisenberg et al., 2009). The numbers used for this calculation were based on the university from which the present study's participants were recruited. This presents a potential limitation as these values cannot be generalized for all universities. The needs of various institutions may vary and incur additional costs; however, these exploratory findings are relevant to understanding both the economic and population health benefits of mindfulness training for university students.

Further limitations of the present study included the question "Would you have been interested in learning about mindfulness during your First Year Experience course?” This question could have potentially predisposed participants towards mindfulness and may have increased social desirability bias. Additionally, the question may have acted as a confounding variable, however, there are a few considerations. Importantly, participants were not primary stakeholders in the decision to implement mindfulness. Each participant had already completed their First Year Experience course, and thus would not be directly affected by the decision to augment curriculum with mindfulness training moving forward. This may have limited the influence of potential predisposition towards mindfulness among participants. Participants were not given any information regarding the definition of mindfulness or mindfulness curriculum. Their responses to this question were based entirely on their previous knowledge and experience of mindfulness. For students lacking prior experience of mindfulness, this question may not be a valid measure of mindfulness acceptability. Future research should seek to understand students' formal mindfulness training and practice while conscientiously avoiding any potential predisposing of participants to a particular outcome. In addition, physiological measures alongside self-reported questionnaires following a mindfulness training period would provide 
more robust results and aid in understanding a potential correlation between formal practice, student mindfulness, and stress. Objectivity of students' responses could be enhanced by including second-person assessments of participants' behavior either from professors, family, or friends (Dvořáková et al., 2017).

A difference must be highlighted between state and trait mindfulness. The FFMQ measures trait, or dispositional, mindfulness. Therefore, one cannot assume correlation between one's intentional mindfulness training and their selfreported dispositional mindfulness. However, Wheeler et al. (2017) noted that mindfulness training induced "learned dispositional" mindfulness in those who, prior to the mindfulness training intervention, did not score highly on the FFMQ. The present study used a sample of convenience, which resulted in a small sample size and lack of sample diversity. Participant recruitment was impacted by campus closure due to the COVID-19 pandemic. COVID-19induced stress may have contributed to the elevated PSS scores reported by the sample.

Another potential limitation, evident in reviewed literature (Watier \& Dubois, 2016; Basso et al., 2018), is the variations which exist in an experimental definition of mindfulness. This hinders the establishment of an operationalized and standardized protocol for mindfulness study (Zhang et al., 2019). An important note in the dissemination of these findings is the distinction between adaptive anxiety and stress, which is intrinsic to the human experience, and maladaptive symptomatology of psychopathology. Mindfulness is not suggested as an alternative to medication or psychotherapy for those diagnosed with a mental health disorder, but rather as an augmentation to universal educational policy and healthcare interventions to promote population health and well-being. One must refer to their clinician or healthcare provider regarding the mental health care protocol best suited to their individual needs (Galante et al., 2018). Finally, research has suggested that the FFMQ lacks cross-cultural validity (Karl et al., 2020). Karl et al. (2020) demonstrated that the FFMQ was a better measure of mindfulness in individualistic cultures (i.e., Western cultures) as compared to non-Western cultures.

Strengths of the present study include the use of reliable and valid measures for participant mindfulness and stress (Bohlmeijer et al., 2011; Baer et al., 2006; Asensio-Martinez et al., 2019; Cohen et al., 1983; Roberti, Harrington, \& Storch, 2006). The negative correlation between FMMQ and PSS scores remained significant at a conservative pvalue cutoff $(<0.01)$ after outliers were removed. This study incorporated literature from a comprehensive and multidisciplinary body of evidence and synthesized perspectives of science and technology, social sciences, and the delivery of efficient and effective health interventions to support well-being. Mindfulness is an accessible and inclusive intervention which may be adapted for diverse student needs and culturally sensitive contexts (Thompson\& Van Vliet, 2018; Masterpasqua, 2012). Although the COVID-19 pandemic may have heightened participant's self- reported stress, a strength lies in the PSS's design as it addresses one's perception of situational circumstances. Individuals undergoing similar life circumstances may still score differently on the PSS due to differences in each individual's perceived stress responses. Finally, the findings discussed in this article have been presented to university stakeholders. This knowledge translation is a strength of the present study, as it is demonstrative of using practical and relevant data to support research-based education and student well-being strategy.

Suggestions for future research include the reproduction of the current study's methodology with the inclusion of a large-scale and diverse sample size. Further demographic data should be collected regarding participant mindfulness experience to understand the intersectional implications of race, sex, education, socioeconomic status, and cultural norms. As this study was exploratory and aimed at examining the feasibility of mindfulness for university students, data was not collected regarding participant's mindfulness training experience. Since mindfulness acceptability was high in the present study, further experimental research should be conducted to produce quantifiable 
metrics regarding the optimal mindfulness training intervention to address student needs. Future research is needed to examine the efficacy and efficiency of a mindfulness training course, with data acquired both pre- and post- training period. The field may be strengthened through the operationalization and standardization of a mindfulness training program for research. This will promote methodological transparency, reproducibility, and robust data acquisition. Collecting longitudinal data may also be beneficial to understanding if students continue to practice mindfulness beyond any formal training and if so, how this continues to impact their mental health.

A difficulty in the field of cognitive neuroscience and clinical psychology is in understanding the correlation between specific brain regions and behavior. It is difficult to precisely identify the neural activation associated with psychopathology. Further large-scale longitudinal research should be conducted, implementing both neuroimaging techniques and biological sample collection to understand the correlation between psychopathology and neuroplastic changes associated with mindfulness training.

\section{RECOMMENDATION}

Universities should augment first-year curriculum with mindfulness training. Students' higher-than-average selfreported perceived stress in the present study is indicative of the need for enhanced mental health strategy. The principles of mindfulness have theoretical basis in cognitive neuroscience and behavioral economics, alongside robust support in current population health research, making mindfulness-based interventions both data-informed and science-driven. Student self-reported acceptability of mindfulness training was high in the present study. Further, findings from the ROI Calculator suggests that mindfulness training for first-year students would result in both added tuition revenue and added lifetime earnings for retained students (Eisenberg et al., 2009).

Regarding surveillance and monitoring, further on-campus research should be conducted to ascertain the most effective and efficient program of mindfulness training relevant to student needs. The results of the present study, discussed in light of previous research, strongly suggest potential for mindfulness training to provide both mental health and economic benefits for students and universities, respectively. 


\section{REFERENCES}

Asensio-Martínez, Á., Masluk, B., Montero-Marin, J., Olivan-Blázquez, B., Navarro-Gil, M. T., García-Campayo, J., \& Magallón-Botaya, R. (2019). Validation of Five Facets Mindfulness Questionnaire-Short form, in Spanish, general health care services patients sample: Prediction of depression through mindfulness scale. Plos one, 14(4), e0214503. https://doi.org/10.1371/journal.pone.0214503

Baer, R.A., Carmody, J., \& Hunsinger, M. (2012). Weekly change in mindfulness and perceived stress in a Mindfulness-Based Stress Reduction Program. Journal of Clinical Psychology, 68: 755-

765. https://doi.org/10.1002/jclp.21865

Baer, R. A., Smith, G. T., Hopkins, J., Krietemeyer, J., \& Toney, L. (2006). Using self- report assessment methods to explore facets of mindfulness. Assessment, 13, 27- 45. https://doi.org/10.1177/1073191105283504

Baer, R. A., Smith, G. T., Lykins, E., Button, D., Krietemeyer, J., Sauer, S., ...Williams, J. M. G. (2008). Construct validity of the Five Facet Mindfulness Questionnaire in meditating and nonmeditating samples. Assessment, 15(3), 329-342. https://doi.org/10.1177/1073191107313003

Baik, S. H., Fox, R. S., Mills, S. D., Roesch, S. C., Sadler, G. R., Klonoff, E. A., \& Malcarne, V. L. (2019). Reliability and validity of the Perceived Stress Scale-10 in Hispanic Americans with English or Spanish language preference. Journal of Health Psychology, 24(5), 628-639. https://doi.org/10.1177/1359105316684938

Basso, J. C., McHale, A., Ende, V., Oberlin, D. J., \& Suzuki, W. A. (2019). Brief, daily meditation enhances attention, memory, mood, and emotional regulation in non-experienced meditators. Behavioural Brain Research, 356, 208-220. https://doi.org/10.1016/j.bbr.2018.08.023

Bohlmeijer, E., Ten Klooster, P.M., Fledderus, M., Veehof, M., Baer, R. (2011). Psychometric properties of the Five Facet Mindfulness Questionnaire in depressed adults and development of a short form. Assessment, 18(3), 308-20. https://doi.org/10.1177/1073191111408231

Bostic, J. Q., Nevarez, M. D., Potter, M. P., Prince, J. B., Benningfield, M. M., \& Aguirre, B. A. (2015). Being present at school: Implementing mindfulness in schools. Child and Adolescent Psychiatric Clinics of North America, 24(2), 245-259. https://doi.org/10.1016/j.chc.2014.11.010

Brown, K. W., \& Ryan, R. M. (2003). The benefits of being present: Mindfulness and its role in psychological wellbeing. Journal of Personality and Social Psychology, 84, 822-848. https://doi.org/10.1037/0022-3514.84.4.822

Charbonneau, D. (2019). Model of mindfulness and mental health outcomes: Need fulfillment and resilience as mediators $=$ Modèle de résultats de la pleine conscience et de la santé mentale: besoin de réalisation et de résilience en tant que médiateurs. Canadian Journal of Behavioural Science / Revue Canadienne Des Sciences Du Comportement, 51(4), 239-247. https://doi.org/10.1037/cbs0000137

Christopher, M. S., Neuser, N. J., Michael, P. G., \& Baitmangalkar, A. (2012). Exploring the psychometric properties of the Five Facet Mindfulness Questionnaire. Mindfulness, 3(2), 124 131. https://doi.org/10.1007/s12671-011-0086-x.

Cohen, S., Kamarck, T., \& Mermelstein, R. (1983). A global measure of perceived stress. Journal of Health and Social Behavior, 24, 385-396. https://doi.org/10.2307/2136404 
Davis, D. M., \& Hayes, J. A. (2012). What are the benefits of mindfulness? Monitor on Psychology, 43(7). https://doi.org/10.1037/a0022062

Deci, E. L., \& Ryan, R. M. (1985). Intrinsic motivation and self-determination in human behavior. New York, NY: Plenum. https://doi.org/10.1007/978-1-4899-2271-7

Dogan, T. (2012). A long-term study of the counseling needs of Turkish university students. Journal of Counseling and Development, 90(1), 91-96. https://doi.org/10.1111/j.1556-6676.2012.00012.x

Duffy, A., Saunders, K. E. A., Malhi, G. S., Patten, S., Cipriani, A., McNevin, S. H., ... Geddes, J. (2019). Mental health care for university students: A way forward? The Lancet Psychiatry, 6(11), 885-887. https://doi.org/10.1016/S2215-0366(19)30275-5

Dunning, D. L., Griffiths, K., Kuyken, W., Crane, C., Foulkes, L., Parker, J., \& Dalgleish, T. (2019). Research review: The effects of mindfulness-based interventions on cognition and mental health in children and adolescents-A meta-analysis of randomized controlled trials. Journal of Child Psychology and Psychiatry, 60(3), 244-258. https://doi.org/10.1111/jcpp.12980

Dvořáková, K., Kishida, M., Li, J., Elavsky, S., Broderick, P., Agrusti, M., \& Greenberg, M. (2017) Promoting healthy transition to college through mindfulness training with first-year college students: Pilot randomized controlled trial. Journal of American College Health, 65(4), 259-267. https://doi.org/10.1080/07448481.2017.1278605

Eisenberg, Daniel, Ezra Golberstein, and Justin B. Hunt. 2009. "Mental Health and Academic Success in College." B.E. Journal of Economic Analysis and Policy, 9(1): Article 40. https://doi.org/10.2202/19351682.2191

Eisenberg, D., Lipson, S. K., \& Posselt, J. (2016). Promoting resilience, retention, and mental health. New Directions for Student Services, 2016(156) 87-95. https://doi.org/10.1002/ss.20194

Farb, N. A., Anderson, A. K., Mayberg, H., Bean, J., McKeon, D., \& Segal, Z. V. (2010). Minding one's emotions: Mindfulness training alters the neural expression of sadness. Emotion (Washington, D.C.), 10(1), 25-33. https://doi.org/10.1037/a0017151

Feliu-Soler, A., Cebolla, A., McCracken, L. M., D’Amico, F., Knapp, M., López-Montoyo, A., ... Luciano, J. V. (2018). Economic impact of third-wave cognitive behavioral therapies: A systematic review and quality assessment of economic evaluations in randomized controlled trials. Behavior Therapy, 49(1), 124-147. https://doi.org/10.1016/j.beth.2017.07.001

Flett, J. A. M., Conner, T. S., Riordan, B. C., Patterson, T., \& Hayne, H. (2020). App-based mindfulness meditation for psychological distress and adjustment to college in incoming university students: a pragmatic, randomised, waitlist-controlled trial. Psychology \& Health, 1-26. https://doi.org/10.1080/08870446.2019.1711089

Galante, J., Dufour, G., Vainre, M., Wagner, A. P., Stochl, J., Benton, A., ... Jones, P. (2018). A mindfulness-based intervention to increase resilience to stress in university students (the Mindful Student Study): A pragmatic randomized controlled trial. The Lancet Public Health, 3(2). https://doi.org/10.1016/s2468-2667(17)30231-1 
Gross, J. J., \& Levenson, R. W. (1997). Hiding feelings: The acute effects of inhibiting negative and positive emotion. Journal of Abnormal Psychology, 106, 95-103. https://doi.org/10.1037/0021-843X.106.1.95

Herman, P.M., Anderson, M.L., Sherman, K.J., Balderson, B.H., Turner, J.A., \& Cherkin, D.C. (2017). Costeffectiveness of mindfulness-based stress reduction versus cognitive behavioral therapy or usual care among adults with chronic low back pain. Spine, 42, 1511-1520. https://doi.org/10.1097/BRS.0000000000002344

Hunt, J., \& Eisenburg, D. (2010). Mental health problems and help-seeking behavior among college students. Journal of Adolescent Health, 46(1), 3-10. https://doi.org/10.1016/j.jadohealth.2009.08.008

Hochster, A., Block-Lerner, J., Marks, D. R., \& Erblich, J. (2018). Mindfulness buffers the effects of cue-induced craving on alcohol demand in college drinkers. Addictive Behaviors, 84, 53-56. https://doi.org/10.1016/j.addbeh.2018.03.013

Karl, J. A., Prado, S. M. M., Gračanin, A., Verhaeghen, P., Ramos, A., Mandal, S. P., ... Fischer, R. (2020). The cross-cultural validity of the Five-Facet Mindfulness Questionnaire across 16 countries. Mindfulness, 11(5), 1226-1237.

Kessler, R.C., Berglund, P., Demler, O., Jin, R., Walters, E.E. (2005). Lifetime prevalence and age-of-onset distributions' of DSM-IV disorders in the national comorbidity survey replication. Archives of General Psychiatry, 62(6), 593-602. https://doi.org/10.1001/archpsyc.62.6.593

Kuyken, W., Nuthall, E., Byford, S, Crane, C., Dalgleish, T., Ford, T., ... Williams, J.M.G. (2017). The effectiveness and cost-effectiveness of a mindfulness training programme in schools compared with normal school provision (MYRIAD): Study protocol for a randomised controlled trial. Trials, 18(1), 194 (2017). https://doi.org/10.1186/s13063-017-1917-4

Kwok, J.M., \& Ng, D.K. (2016). A Study of the Perceived Stress Level of University Students in Hong Kong. International Journal of Psychological Studies, 8, 91. https://doi.org/10.5539/ijps.v8n4p91

Lavelle Heineberg, B. D. (2016). Promoting caring: Mindfulness- and compassion-based contemplative training for educators and students. In K. A. Schonert-Reichl \& R. W. Roeser (Eds.), Handbook of mindfulness in education: Integrating theory and research into practice. (pp. 285-294). Springer-Verlag Publishing. https://doi.org/10.1007/978-1-4939-3506-2

Lee, E. H. (2012). Review of the psychometric evidence of the perceived stress scale. Asian nursing research, 6(4), 121-127. https://doi.org/10.1016/j.anr.2012.08.004

Lipson, S. K., Abelson, S., Ceglarek, P., Phillips, M., \& Eisenberg, D. (2019). Investing in student mental health. American Council on Education (Washington, D.C.).

Masterpasqua, F. (2012). Making mindfulness accessible. PsycCRITIQUES, 57(40). https://doi.org/10.1037/a0029908

Medvedev, O. N., Krägeloh, C. U., Hill, E. M., Billington, R., Siegert, R. J., Webster, C. S., ... Henning, M. A. (2019). Rasch analysis of the Perceived Stress Scale: Transformation from an ordinal to a linear measure. Journal of Health Psychology, 24(8), 1070-1081. https://doi.org/10.1177/1359105316689603 
Meiklejohn, J., Phillips, C., Freedman, M. L., Griffin, M. L., Biegel, G., Roach, A., ... Saltzman, A. (2012). Integrating mindfulness training into K-12 education: Fostering the resilience of teachers and students. Mindfulness, 3(4), 291-307. https://doi.org/10.1007/s12671-012-0094-5

Minkler, T.O., Glass, C.R., \& Hut, M (2020) Mindfulness training for a college team: Feasibility, acceptability, and effectiveness from within an athletic department. Journal of Applied Sport

Psychology. https://doi.org/10.1080/10413200.2020.1739169

Nunnally, J. C., \& Bernstein, I. H. (1994). Psychometric theory (3rd ed.). New York :McGraw-Hill. ISBN: 007047849x.

Riet, P. V. D., Levett-Jones, T., \& Aquino-Russell, C. (2018). The effectiveness of mindfulness meditation for nurses and nursing students: An integrated literature review. Nurse Education Today, 65, 201-211. https://doi.org/10.1016/j.nedt.2018.03.018

Roberti, J., Harrington, L., \& Storch, E. (2006). Further psychometric support for the 10-item version of the perceived stress scale. Journal of College Counseling, 9(2), 135-147. https://doi.org/10.1002/j.2161-1882.2006.tb00100.x

Ryan, R. M., \& Deci, E. L. (2000). Self-determination theory and the facilitation of intrinsic motivation, social development, and well-being. American Psychologist, 55, 68-78. https://doi.org/10.1037/0003-066X.55.1.68

Schultz, P. P., \& Ryan, R. M. (2015). The "Why", "What", and "How" of healthy self- regulation: Mindfulness and well-being from a self-determination theory perspective. In B. D. Ostafin, et al. (Eds.), Handbook of mindfulness and self-regulation (pp. 81-94). New York: Springer. https://doi.org/10.1007/978-1-4939-2263-5

Shallcross, A.J., Lu, N.Y. \& Hays, R.D. (2020). Evaluation of the Psychometric Properties of the Five Facet of Mindfulness Questionnaire. Journal of Psychopathology and Behavioral Assessment, 1-10. https://doi.org/10.1007/s10862-019-09776-5

Shapiro, S.L., Schwartz, G.E., \& Bonner, G. (1998). Effects of mindfulness-based stress reduction on medical and premedical students. Journal of Behavioral Medicine, 21(6), 581-99. https://doi.org/10.1023/A:1018700829825

Taylor, B. L., Strauss, C., Cavanagh, K., \& Jones, F. (2014). The effectiveness of self-help mindfulness-based cognitive therapy in a student sample: A randomised controlled trial. Behaviour Research and Therapy, 63, 6369. https://doi.org/10.1016/j.brat.2014.09.007

Teixeira, P. J., Marques, M. M., Silva, M. N., Brunet, J., Duda, J. L., Haerens, L., ...Hagger, M. S. (2020). A classification of motivation and behavior change techniques used in self-determination theory-based interventions in health contexts. Motivation Science. https://doi.org/10.1037/mot0000172

Thompson, K., \& Van Vliet, P. (2018). Critical reflection on the ethics of mindfulness. Australian Social Work, 71(1), 120-128. https://doi.org/10.1080/0312407X.2017.1364396

Watier, N., \& Dubois, M. (2016). The Effects of a brief mindfulness exercise on executive attention and recognition memory. Mindfulness, 7(3), 745-753. https://doi.org/10.1007/s12671-016-0514-z

Wheeler, M. S., Arnkoff, D. B., \& Glass, C. R. (2017). The neuroscience of mindfulness: How mindfulness alters the brain and facilitates emotion regulation. Mindfulness, 8(6), 1471- 1487. https://doi.org/10.1007/s12671-017-0742-x 
Wolkin, J. (2015) Cultivating multiple aspects of attention through mindfulness meditation accounts for psychological well-being through decreased rumination. Psychology Research and Behavior Management, 8, 171-180. https://doi.org/10.2147/PRBM.S31458

Zhang, Q., Wang, Z., Wang, X., Liu, L., Zhang, J., \& Zhou, R. (2019). The Effects of Different Stages of Mindfulness Meditation Training on Emotion Regulation. Frontiers in Human Neuroscience, 13. https://doi.org/10.3389/fnhum.2019.00208

Address author correspondence to:

Christina Maher

Fairfield University

Integrated Nursing and Health Studies Initiative (c/o Dr. Patrick Kelley)

1073 N Benson Rd

Fairfield, CT 06824

christina.maher@student.fairfield.edu

\section{Author's Note and Acknowledgements}

The author would like to recognize and thank Dr. Patrick Kelley (Fairfield University) for insightful discussion, comments on the manuscript, and support. 